\title{
Observational Study
}

\section{e Clinical Outcome Following Radiofrequency Denervation for Refractory Sacroiliac Joint Dysfunction Using the Simplicity III Probe: A 12-Month Retrospective Evaluation}

Dominic Hegarty, PhD, FCA RSCI

From: Cork University Hospital, Cork, Ireland

Address Correspondence: Dominic Hegarty, PhD, FCA

$\mathrm{RSCl}$

Consultant Pain Management \& Neuromodulation, Cork University Hospital Honorary Consultant Guy's \& St. Thomas' Hospital, London Clinical Lead Neuromodulation Research, Tyndall National Institute, UCC MCN: 21473 E-mail: dominichegarty@hotmail.com

Disclaimer: There was no external funding in the preparation of this manuscript.

Conflict of interest: Each

author certifies that he or

she, or a member of his or her immediate family, has no commercial association (i.e., consultancies, stock ownership, equity interest, patent/licensing arrangements, etc.) that might

pose a conflict of interest in connection with the submitted manuscript.

Manuscript received: 05-01-2015 Revised manuscript received: o6-28-2018

Accepted for publication: 07-29-2015

Free full manuscript: www.painphysicianjournal.com
Background: Sacroiliac joint syndrome (SIJ) is diagnosed in $10 \%$ to $25 \%$ of cases of lower back pain. The response to traditional radiofrequency (RF) denervation of the SIJ has being inconsistent. The Simplicity III RF probe (Neruotherm. Inc.) offers a novel treatment option.

Objective: To evaluate the long-term clinical outcome (12 months) refractory SIJ syndrome in terms of pain intensity and functional improvement. A $50 \%$ reduction in intensity pain intensity (VAS) at 12 months was deemed clinically significant.

Study Design: A 12-month retrospective observational evaluation all of adults treated with RF for refractory SIJ.

Setting: Chronic pain management center.

Methods: The medical records of all adults treated with this technique was retrospectively reviewed. The primary outcome was pain intensity scores (VAS) over a 12 months period; Secondary outcomes included Roland-Morris Functional scores (RMF), Brief Pain Inventory (BPI), general health assessment (Sf12), and patient satisfaction scores (GPI), which were recorded pre and post denervation.

Results: Pain Intensity improved by 4.7 points compared to pre-treatment representing a $61 \%$ reduction in pain at 12 months $(n=11, P<0.001)$. Significant improvements in (a) RMF $(P<$ $0.01, \mathrm{~W}^{2}=0.63$ (large effect size); (b) BPI $\left(P<0.001, \mathrm{~W}^{2}=0.72\right.$ (strong effect size); and (c) $\mathrm{Sf1} 2(P<0.01)$ were noted. Overall patients were satisfied with the outcome $(\mathrm{GPI}=77.7 \%)$.

Limitations: The retrospective in nature of the study and the small sample size are limitations. As it was our policy to monitor the progress of the individuals since the introduction of this technique a reliable method of recording the baseline and outcome variables at each point of contact was in place. Access to a complete set of variables in all individuals over a 12-month period was therefore possible, which we feel contributes to the quality of the dataset.

Conclusion: By creating a consistent radiofrequency lesion between the sacral foramen and the SIJ will reliably capture the innervation to the SIJ with significant long-term clinical improvement. This technique should be considered earlier in the treatment algorithm of individuals suffering from SIJ symptoms.

Key words: Sacroiliac Joint syndrome, radiofreqency denervation, Simplicity III probe, chronic pain, long-term clinical outcome, Roland-Morris Functional scores (RMF), Brief Pain Inventory (BPI), general health assessment (Sf12), patient satisfaction scores (GPI)

Pain Physician 2016; 19:E129-E135 
S acroiliac joint syndrome (SIJ) has been implicated as the primary source of pain in $10 \%$ to $25 \%$ of cases of lower back pain (1). It results in clinical consequences such as constant pain, sleep disturbance, and functional disability. Pharmacological therapy is often of limited benefit and although peri-articular local-anesthetic injections may offer relief, some individuals fail to respond (2).

Radiofrequency denervation (RF) of the SIJ has been proposed as a long-lasting treatment option for patients who report temporary pain relief with 2 sets of peri-articular local-anesthetic injections. However, the response to RF treatment of the SIJ has been inconsistent probably due to (a) the diffuse innervation of the SIJ extending from the L5 to S4; (b) the variety of RF techniques used; and (c) the limits imposed by the equipment design $(3,4)$.

\section{Objective}

To evaluate the long-term clinical outcome (12 months) following the use of the Simplicity III RF probe in those individuals refractory to conventional SIJ management in terms of pain intensity and functional improvement.

\section{Methods}

Using a retrospective observational study design, the medical records of all individuals who had RF denervation of the SIJ using the Simplicity III RF probe (Neurotherm Inc.) in the previous 12 months were examined.

In keeping with the International Association for the Study of Pain (IASP) criteria, refractory SIJ dysfunction was defined as individuals who complained of persistent lower back pain but who had received $>50 \%$ symptom relief from 2 or more diagnostic SIJ blocks performed at 12-week intervals $(5,6)$. A standardized diagnostic corticosteroid/local anesthetic SIJ injection was performed by the same pain physician, using fluoroscopically guided needle positioning on all occasions and the response documented at follow-up.

The medical records were examined using the following inclusion criteria:

A. Adult patients (> 18 years) presenting with predominantly axial pain below the L5 vertebrae lasting longer than 6 months with a positive compression test (such as the Patricks' Test).

B. Confirmed positive response (> 50\% reduction in pain intensity) to 2 or more diagnostic SIJ blocks performed at 12-week intervals using a standard- ized diagnostic corticosteroid/local anesthetic SIJ injection.

C. An average daily Numerical Rating Score (NRS) pain intensity score $>6 / 10$ despite comprehensive nonoperative treatments, including but not limited to activity alteration, non-steroidal anti-inflammatory, physical, and/or manual therapy.

D. Other possible sources of low back pain were reasonably excluded (by means of physical exam, medical history, and magnetic resonance imaging/computed tomography/x-ray, and rheumatology screening as required), including but not limited to bone fractures, the hip joint, symptomatic spondylolisthesis, tumor, and other regional soft tissue structures.

Exclusion criteria included malignant disease, mental handicap, allergy to local anesthetics, pain as a result of peripheral ischemia, and previous SIJ fusion. Patients with a history of potentially confounding intervertebral disc disease or facet joint pain were excluded, unless they had responded well to treatment for these conditions for over 6 months.

\section{Outcome Measures}

The primary outcome measure was mean change from baseline in pain relief, assessed using visual analogue pain scores (VAS) over a 12-month period. A $50 \%$ reduction in pain was deemed clinically significant.

Secondary outcome measures included functionality and quality of life scores (Brief Pain Inventory, Roland-Morris Functional scores), general health assessment (Sf12), and patient satisfaction scores. Patients were requested to report any adverse effects to the physician at each assessment.

\section{Radiofrequency Denervation Technique}

All cases had a standardized radiofrequency (RF) treatment in the prone position using customary aseptic techniques. RF denervation was undertaken as a day-case procedure and involved:

\section{(a) L5 primary dorsal ramus RF lesion}

The C-arm is positioned for anterior-posterior (AP) and caudal tilt projection. The sulcus between the S1 superior articular process and the sacral ala are identified. A 18 gauge, $100 \mathrm{~mm}$ long, $10 \mathrm{~mm}$ straight sharp RF cannula (Neurotherm, Inc.) was placed to lie in contact with the S1 superior articular processes just above the groove formed between superior articular process and the sacral ala. Appropriate positioning is confirmed us- 
ing the AP, ipsilateral, oblique, and lateral views. Motor stimulation is carried out at $2 \mathrm{~Hz}$ and at up to $1.5 \mathrm{~V}$ verifying no motor recruitment in the ipsilateral lower extremity. A $2 \%$ lidocaine in solution $(5 \mathrm{~mL})$ is injected through the cannula, and RF lesioning of the L5 dorsal root is performed at $85^{\circ} \mathrm{C}$ for up to 90 seconds. Subsequently local anesthesia $(0.5 \%$ bupivacaine and $10 \mathrm{mg}$ methylprednisolone steroid) is injected through the cannula after the lesion is complete.

\section{(b) SIJ denervation}

The C-arm is rotated between the AP, lateral, and oblique views to position the device correctly. The percutaneous entry point was identified $1 \mathrm{~cm}$ lateral of and below the $\mathrm{S} 4$ foramen. Using a $100 \mathrm{~mm}$ needle, and under fluoroscopic guidance, the proposed track is anasthetized using $2 \%$ lidocaine with adrenaline $(7 \mathrm{mg} / \mathrm{kg})$. This needle position is then used to guide the correct positioning of the Simplicity III device in terms of angulation, depth, and direction.

Under fluoroscopic guidance the probe was advanced along this pre-anasthetized track device following the curvature of the sacrum to the sacral ala (Fig. 1 \& Fig. 2). Ideally the 3 active contacts are on the appropriate position to legion the lateral branches of S1, S2, S3, and S4, respectively. The final position of the electrode should lie:

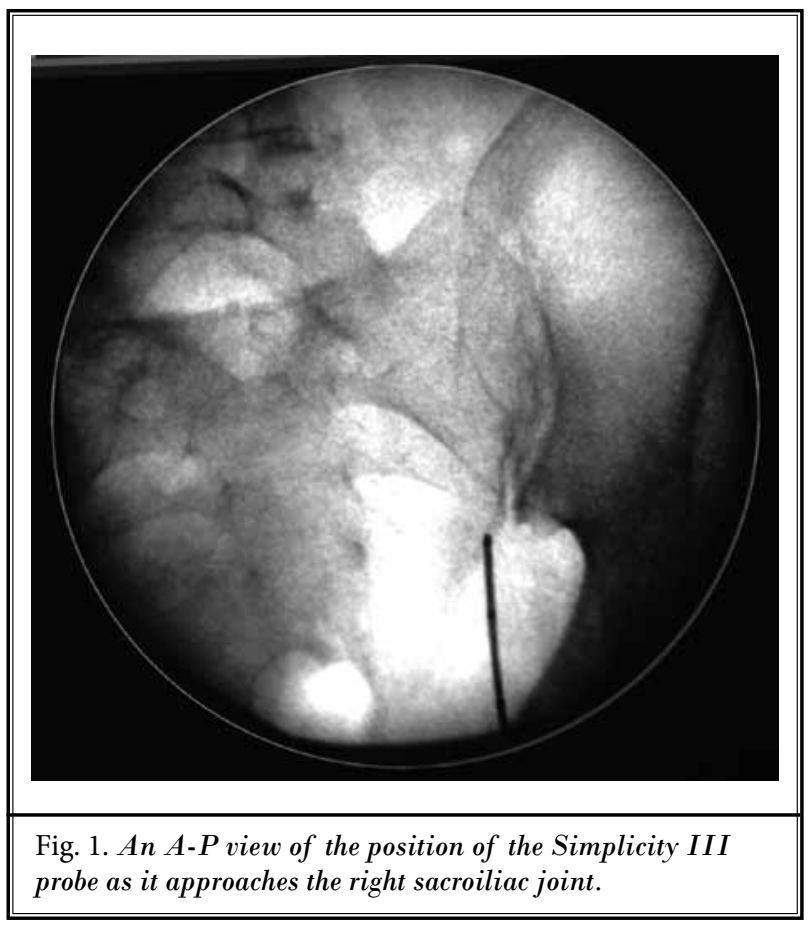

A. Lateral to the foramen and medial to the SIJ in the AP position (Fig. 2). The device should not extend beyond the sacral ala and it should lie no more the $1.5 \mathrm{~cm}$ above the periosteum in the lateral position. This positioning ensures that the procedure is tolerated by the awake patient and minimizes the post-operative pain that has previously reported.

B. Safe distance from the dermis to prevent skin injury, however, direct contact with the sacral periosteum is not required (Fig. $3 a$ \& $3 b$ ). As a guide, the maximum distance from the sacral periosteum device in the lateral position should be half way between the skin and the periosterum.

Denervation is then carried out using the preprogrammed RF sequence ( $89^{\circ} \mathrm{C}$ for 8 minutes in total). On completion $30 \mathrm{mg}$ of methylprednisolone and $0.5 \%$ of bupivacaine ( $3 \mathrm{mg} / \mathrm{kg}$, up to $10 \mathrm{~mL}$ ) are injected to prevent and reduce the occurrence of neuritis.

\section{Post-procedural Instructions}

All individuals were advised regarding the risk of post procedural pain and analgesics were prescribed in anticipation of this issue. In addition all individuals were encouraged to undertake daily physiotherapy ex-

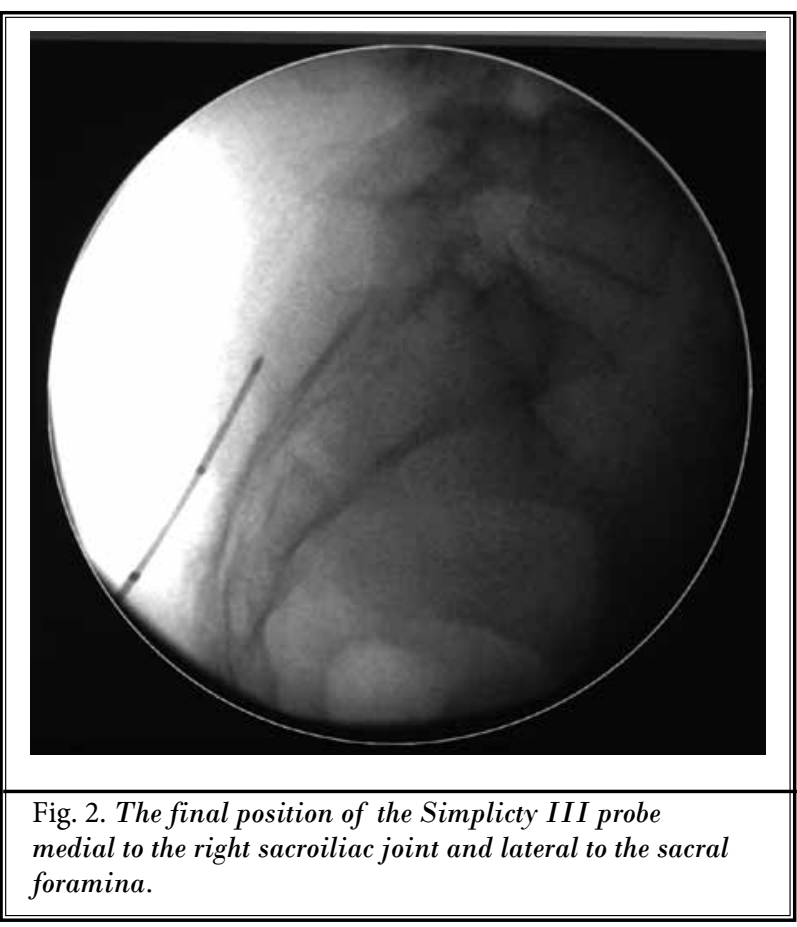




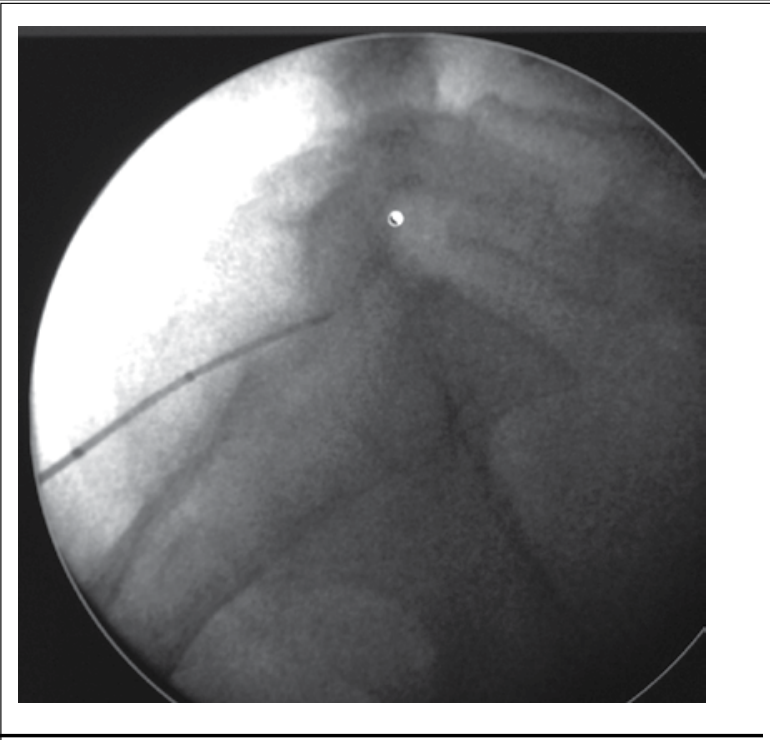

Fig. 3. The lateral view of initial position and final position of the Simplicity III probe.
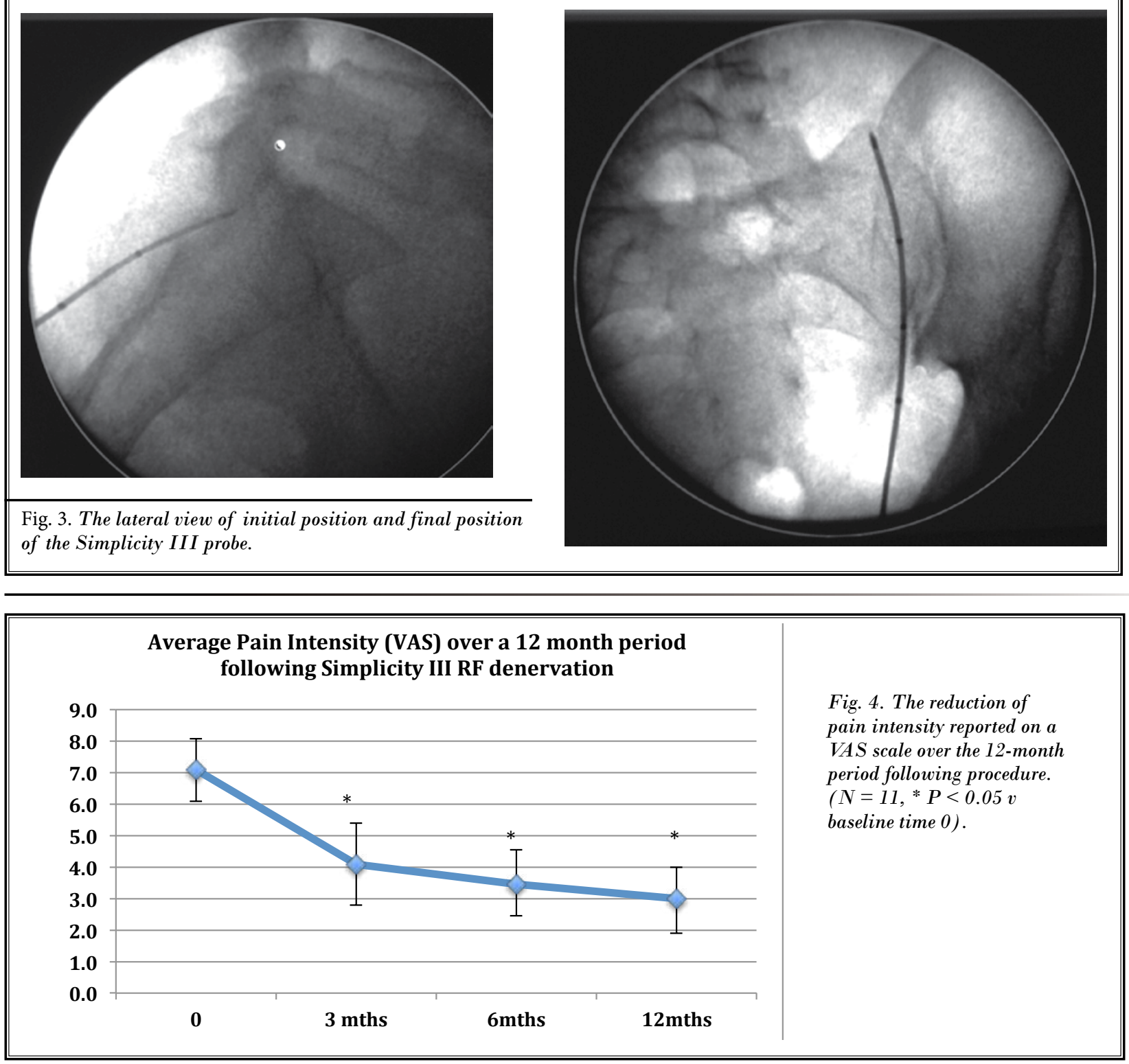

ercises targeting the SIJ and were provide with information booklets regarding 3 suitable exercises.

\section{Statistical Analysis}

All data was recorded using Microsoft Excel (Mac 2010) and analyzed using StatMac:plus v5 (2010, AnalystSoft Inc., 2014).

\section{Results}

Denervation of the SIJ using this technique was performed in 11 women (average age $55.2 \pm 13.9$ years (range 35.5 - 75 years) with an average refractory SIJ dysfunction period of $17.5 \pm 4.2$ months (range $12-24$ months). The mean pre-procedural NRS pain intensity score was $7.7 \pm 1.0$ (range $6-9$ ). All cases had unilateral refractory SIJ dysfunction (6 right sided and 5 left sided).

\section{Pain Intensity Score}

A mean reduction of 4.7 points was noted in the VAS pain intensity over 12 months. This represented a mean improvement of $61 \%$ in pain intensity. The VAS score declined steadily over the study period as illustrated in Fig. 4. ANOVA analysis showed that in the 
Table 1. The ANOVA analysis for pain intensity (Visual Analogue Score (VAS), Roland Morris Functional score (RMF), and general health score (SF12, Student t-test) before and 12 months following the simplicity III RF procedure (all mean, $(S D), n=11$ unless indicated).

\begin{tabular}{|c|c|c|c|c|}
\hline Variable & Pre-procedure & 12 months follow up & F-Ratio (effect size) & $P$ value \\
\hline \multicolumn{5}{|l|}{$\begin{array}{l}\text { Pain Intensity } \\
\text { VAS }(0-10 \mathrm{~cm})\end{array}$} \\
\hline Average & $7.7(1.0)$ & $3.0(1.0)$ & $40.9(.73)$ & $<0.001$ \\
\hline At Rest & $6(1.1)$ & $2.6(0.5)$ & $12.8(.49)$ & $<0.001$ \\
\hline On Movement & $8.4(1.1)$ & $3.5(0.7)$ & $14.5(.52)$ & $<0.001$ \\
\hline \multicolumn{5}{|l|}{ RMF Score } \\
\hline & $12.9(2.8)$ & $5.7(1.4)$ & $22(.63)$ & $<0.01$ \\
\hline Sf12 & & & & Student T-test \\
\hline Physical & $20.3(1.8)$ & $41.0(3.1)$ & (d.f 10) & $<0.001$ \\
\hline Mental & $40.2(1.4)$ & $45.7(5.0)$ & (d.f 10) & 0.01 \\
\hline
\end{tabular}

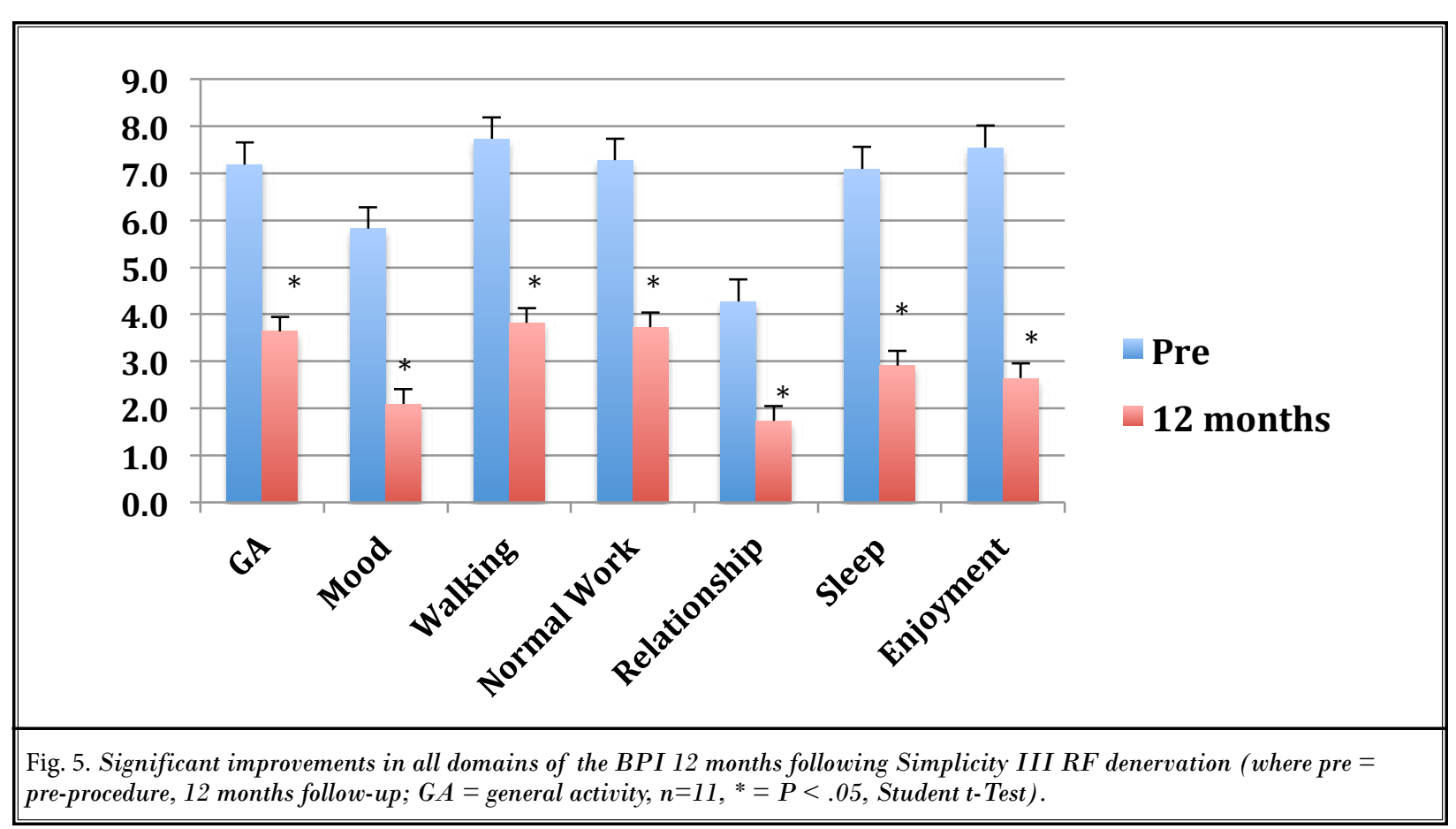

12-month period following treatment the average daily pain intensity, pain at rest, and on movement all significantly improved following treatment $(56-61 \%$ improvement) (Table 1). Post hoc analysis identified a significant improvement in pain scores at both the 3 and 6 month assessments (Tukey HSD Test for Differences Between Means $P=.0001$ ).

\section{Functionality Score}

Following treatment the RMF functionality score continued to show a significant improvement at 12 months compared to the pre-procedural scores (ANOVA, F-ratio [4,50] 22.0, $P<0.01, W^{2}=0.63$ [large effect size]). Post hoc analysis identified that within 3 months of the procedure, a significant improvement in functionality was achieved $(P<.001)$ and this was maintained for the remainder of the assessment period.

\section{Brief Pain Inventory}

ANOVA analysis of the mean BPI scores showed significant improvement over the 12 month follow up period: $F(3,40)=41.9 . P<0.001, W^{2} 0.72$ (strong effect 
size). Fig. 5 illustrates the mean change in each domain of the BPI over 12 months.

\section{General Health Score: Sf12}

The pre-procedural Sf12 scores in both the physical component capacity and mental component scores were noted to be lower than the expected norm. However, at 12 months following RF treatment, both showed a significant improvement (T-test, $P<0.01$, Table 1).

\section{Global Patient Improvement Assessment}

Twelve months after treatment all patients reported a positive improvement of $77.7 \%$ in their quality of life, ranging from $70 \%$ to $85 \%$ improvement.

\section{Adverse Events}

The most common issue for all individuals was post procedural pain that required regular analgesic management for between 10 and 14 days. Despite all individuals being advised about the need for a post procedure analgesic treatment, this still was problematic for some individuals.

One individual complained of "burning sensation" in the skin area overlying the SIJ after 3 months. This responded to the subcutaneous infiltration of $10 \mathrm{~mL}$ of $0.25 \%$ bupivacaine with 40 mgs of methylprednisolone (depo-medrone [Pharmacia]) under ultrasound guidance combined with 12 weeks transdermal treatment lignocaine patches. The symptoms had decreased by $70 \%$ at the 12-month follow-up.

Two individuals noted paraesthesia in a circular area overlying the SIJ when directly asked. This was confirmed with von Frey testing to the region. It was not problematic for the individuals concerned and they did not require any intervention.

One individual required an additional lumbar L5/ S1 facet joint injection on the ipsilateral side to the treated SIJ 6 months after the denervation to control ongoing "lower back pain" (pain VAS intensity 5/10). Three months follow-up confirmed a positive response to this additional injection. The SIJ pain remained under control.

\section{Discussion}

This study highlights the clinical efficacy of the Simplicity III RF probe device in this treatment of for refractory SIJ syndrome. Twelve months after the procedure the average reduction in pain was $61 \%$; there was also a significant improvement in an array of clinically relevant secondary outcomes with minimal adverse ef- fects. However, as this procedure is more invasive than other treatment options, we propose that the Simplicity III RF probe should be considered as a suitable technique for SIJ cases refractory to conservative and other interventions.

The SIJ is a diarthrodial synovial joint where only the anterior part is a true synovial joint. The posterior part is a syndesmosis consisting of the ligamenta sacroiliaca, the musculus gluteus medius and minimus, and the musculus piriformis (7). The SIJ is innervated mainly by the sacral rami dorsales from L5 to S4 as part of a posterior sacral network (PSN) (8). Cadaveric studies have shown that S1-S2 always contributes, and the S3, L5, and S4 contribute $88 \%, 8 \%$, and $4 \%$ to the innervation, respectively (2).

While the cause of SIJ pain can be divided into intra-articular causes (infection, arthritis, spondyloarthropathies, malignancies) and extra-articular causes (fractures, ligamentous injuries, and myofascia), frequently no specific cause can be identified. Currently there are no definite conservative, interventional, or surgical options for the management of SIJ syndrome.

RF denervation has shown to be clinically effective in the management of many pain conditions. While denervation of the SIJ is well-reported (2), reliable outcome after denervation of the SIJ has been problematic because of (a) the diffuse innervation of the SIJ extending from the L5 to $\$ 4$ and (b) by the inconsistency of the techniques used to preform RF at the SIJ.

The design of the probe seeks to remove the technical restrictions imposed by using traditional RF techniques. Firstly, the single percutaneous entry point allows for the efficient and the safe placement of the device under fluoroscopy. This eradicates the need for multiple needle placements conventionally required which reduces the procedure time and improves patient safety. Secondly, the creation of a consistent, broad lesion is possible by systemically applying continuous RF energy $\left(89^{\circ} \mathrm{C}\right)$ to the lateral branches of the S1, S2, S3, and S4 nerves. This offers the clinician an opportunity to provide an effective zone of denervation to the joint. We propose that the ability to provide this denervation zone explains the long-term benefit associated with RF treatment. Thirdly, the pre-programmed energy sequence improves the efficiency of the procedure.

Limitations of our study include the small sample size and the retrospective nature of the study. As it was our policy to monitor the progress of the individuals since the introduction of this technique, a reliable method of recording the baseline and outcome 
variables at each point of contact was in place. Access to a complete set of variables in all individuals over a 12-month period was therefore possible, which we feel contributes to the quality of the dataset.

Although only 11 individuals were included in this study, this represented a cohort that was known to be refractory to all other treatments. This may explain the significant improvements noted in the early phase of treatment (> $50 \%$ within 3 months), nonetheless, the continued improvement over time should be noted. The true incidence of refractory SIJ cases is unknown and needs to be established; future studies could establish this.

\section{Conclusion}

In conclusion this study identified that creating a consistent RF lesion between the sacral foramen and the SIJ will reliably and safely capture the innervation of the SIJ with significant long-term clinical improvement. Based on these results, we propose that the use of this technique should be considered sooner in the treatment of refractory SIJ syndrome.

\section{Acknowledgement}

DH wishes to acknowledge the assistance of Dr. Neil O'Donovan, Consultant Radiologist, Department of Radiology, South Infirmary \& Victoria University Hospital, Cork, Ireland, for preparing the radiological images. I wish to acknowledge the fact our department received an educational grant from PEI Ireland who were the supplier of Neurotherm Equipment to our hospital in the past.

\section{References}

1. Rupert MP, Lee M, Manchikanti L, Dafta $S$, Cohen SP. Evaluation of sacroiliac joint interventions: A systemic appraisal of the literature. Pain Physician 2009; 12:399-418.

2. Hansen H, Manchikanti L, Simopoulos T. A systemic evaluation of the therapeutic effectiveness of sacroiliac joint interventions. Pain Physician 2012; 15:E247E278.

3. Cohen SP, Strassels SA, Kurihara C, Crooks MT, Erdek MA, Forsythe A, Marcuson M. Outcome predictors for sacroiliac joint (lateral branch) radiofrequency denervation. Reg Anesth Pain Med 2009; 34:206-214.

4. Aydin Sm, Gharibo CG, Mehnert M, Stitik TP. The role of radiofrequency ablation for sacroiliac joint pain: A metanalysis. PM R 2010; 2:842-851.

5. Merskey $H$, Bogdok N. Sacroiliac joint pain in Group XXVII: Sacral spinal or a radicular pain syndrome. In: Classification of Chronic Pain: Descriptions of Chronic Pain Syndromes and Definition of Pain Terms. 2nd ed. Task Force on Taxonomy of the international Association for the study of pain. IASP Press, Seattle, 1994, pp 190-119.
6. Hansen HC, Mckenzie-Brown AM, Cohen SP, Swicegood JR, Colson JD, Manchikanti L. Sacroiliac joint interventions: A systemic review, Pain Physician 2007; 8:115-125.

7. Vanelderan P, Szadek K, Cohen SP. Sacroiliac joint pain. Pain Practice 2010; 10:470-478.

8. Roberts SL, Burnham RS, Ravichandiran $\mathrm{Km}$ Agur A, Loh EY. Cadaveric study of sacroiliac joint innervation: Implications for diagnostic blocks and radiofrequency ablation. Reg Anesth Pain Med 2014; 39:456-464. 
